\title{
Protein-Labeling Effects in Confocal Laser Scanning Microscopy
}

\author{
Christopher A. Teske, Magnus Schroeder, Robert Simon, and Juirgen Hubbuch* \\ Institut für Biotechnologie 2, Forschungszentrum Jülich, Jülich 52425, Germany \\ Received: February 9, 2005; In Final Form: April 18, 2005
}

\begin{abstract}
Confocal laser scanning microscopy (CLSM) is being increasingly used for observing protein uptake in porous chromatography resins. Recent CLSM studies have revealed the possible existence of a nondiffusive protein transport mechanism. Observing protein uptake with CLSM requires labeling the protein with a fluorescent probe. This study examines the effect of the probe identity on the subsequent CLSM adsorption profiles. The adsorption of lysozyme conjugated with different fluorescent probes (Cy5, BODIPY FL, Atto 635, and Atto 520) on SP Sepharose Fast Flow was measured using CLSM and zonal chromatography experiments. Results from zonal chromatography show that the retention time of lysozyme-dye conjugates differ significantly from unlabeled lysozyme. The change in retention of lysozyme upon conjugation with a fluorescent probe is consistent with the difference in net charge between the lysozyme-dye conjugate and unlabeled lysozyme. The adsorption profiles measured by CLSM show significantly different behavior depending upon whether the lysozyme-dye conjugate is retained longer or shorter than the unlabeled lysozyme. These results strongly suggest that the lysozyme concentration overshoot observed in previous CLSM experiments is the result of displacement of weaker binding labeled lysozyme by stronger binding unlabeled lysozyme.
\end{abstract}

\section{Introduction}

Confocal laser scanning microscopy (CLSM) has emerged as a powerful method in the study of mass transport and adsorption of proteins in chromatographic stationary phases. ${ }^{1-5}$ If a number of key assumptions are met, CSLM allows the determination of the adsorbed protein concentration within a stationary phase particle as a function of time. This previously inaccessible data provides new information for testing models of protein transport and adsorption in packed beds. Already, CLSM has revealed interesting phenomena in stationary phase particles, such as the existence (under certain conditions) of an internal maximum in the protein concentration as a function of radial position in the particle. ${ }^{4,5}$ Dziennik et al. ${ }^{4}$ and Liapis et al. ${ }^{6}$ attempted to predict the observed concentration overshoots by incorporating an electrophoretic contribution to protein transport. The model considered by Dziennik et al. ${ }^{4}$ predicts an overshoot in the protein pore concentration, but not in the total protein concentration. The model of Liapis et al. ${ }^{6}$ does predict an overshoot in the total protein concentration, but the calculated profiles are not very similar to the experimentally observed profiles by CLSM, and it has been pointed out by Dziennik et al. ${ }^{4}$ that the diffusivity and adsorption isotherm parameters employed are unrealistic.

Use of CLSM requires labeling the protein with a fluorescent probe. With CLSM, only the adsorption and transport of the labeled protein species is observed. Prior to an experimental run, labeled protein is diluted with a large amount of unlabeled protein (typically giving a final dye:protein molar ratio of 1:100 to $1: 20)$ to avoid inner filter and quenching effects. Thus, experimentally, a mixture of labeled and unlabeled proteins is contacted with stationary phase particles and the radial adsorption profile of the labeled protein is observed over time under the microscope.

\footnotetext{
* Corresponding author. E-mail: j.hubbuch@fz-juelich.de.
}

During the labeling reaction, different protein-dye conjugates are likely formed varying in the number of dye molecules attached and the location of those dye molecules on the surface of the protein. ${ }^{7,8} \mathrm{An}$ implicit assumption made when interpreting all CLSM results has been that labeled and unlabeled proteins behave identically. In that case, the fluorescent label only serves as a probe to visualize the movement of protein in the stationary phase. However, if conjugation of the protein with a fluorescent label would change the affinity for the stationary phase or the protein's transport, then in reality, the experiment is a multicomponent system, and interpretation of CLSM results becomes more complicated.

Lewus et al. ${ }^{9}$ have carried out batch and shallow-bed twocomponent protein uptake experiments and shown how the steric mass action (SMA) isotherm ${ }^{10}$ can predict displacement of weaker binding protein by a stronger binding protein. Such an experimental system would show radial concentration humps which are qualitatively similar to those seen in "singlecomponent" CLSM experiments. ${ }^{11}$ Gallant ${ }^{12}$ has also published a computational study calculating radial concentration profiles of two protein components in a single ion-exchange particle with adsorption described by the SMA isotherm. These calculations are also capable of producing a radial hump which is qualitatively similar to those observed in CLSM experiments. The key question of this study is how much the presence of the fluorescent label affects adsorption of the protein and whether such an effect is capable of producing radial humps similar to those observed in other published studies.

The size, hydrophobicity and charge of the fluorescent label are properties which could influence the retention time of the dye-protein conjugate. For CLSM measurements on stationary phases used in hydrophobic interaction chromatography, the hydrophobicity of the dye is likely an important factor in determining how similarly unlabeled and labeled protein molecules behave. Recent work has characterized the hydrophobicity of some commonly used fluorescent dyes ${ }^{13}$ which may 
TABLE 1: Properties of Fluorescent Labels

\begin{tabular}{lcccccc}
\hline \multicolumn{1}{c}{ dye } & MW & $\lambda_{\text {ex }}(\mathrm{nm})$ & $\lambda_{\text {em }}(\mathrm{nm})$ & $\epsilon\left(\lambda_{\max }\right)$ & CF & $z$ \\
\hline Cy5 & 792 & 649 & 670 & 250000 & 0.05 & -1 \\
Atto 635 & 628 & 635 & 659 & 120000 & 0.10 & +1 \\
Atto 520 & 564 & 525 & 545 & 110000 & 0.14 & +1 \\
BodipyFL & 502 & 504 & 512 & 68000 & 0.04 & 0
\end{tabular}

be useful in selecting an appropriate label. Retention time in ion exchange chromatography depends principally on protein charge. Because most commonly used dyes for labeling proteins are charged, an important experiment before using CLSM is to determine to what extent the presence of the label affects the retention time. This study examines the differences in affinity between labeled and unlabeled lysozyme toward the cationexchange resin SP Sepharose Fast Flow and the effects of differences in affinity on the subsequent intraparticle radial concentration profiles measured by CLSM.

\section{Experimental Section}

2.1. Buffer and Protein Solution Preparation. Phosphate buffers were prepared by dissolving sodium dihydrogen phosphate and disodium hydrogen phosphate (Merck, Darmstadt, Germany) in the necessary ratio to give $\mathrm{pH} 7$ with total phosphate as indicated. $6 \mathrm{mM}$ ionic strength solutions in CLSM experiments contained $3 \mathrm{mM}$ phosphate; all other ionic strengths contained $10 \mathrm{mM}$ phosphate. The ionic strength of the solutions was adjusted to the indicated value by adding sodium chloride (KMF, Lohmar, Germany) to the buffer. Carbonate buffer used in the conjugation reaction was prepared by dissolving sodium carbonate (Merck) at $100 \mathrm{mM}$ and then adjusting the necessary $\mathrm{pH}$ with $\mathrm{HCl}$ or $\mathrm{NaOH}$.

Protein solutions were prepared by dissolving lysoyzme (L6876, Sigma-Aldrich, Steinheim, Germany) or $\alpha$-lactalbumin (L-6010, Sigma-Aldrich) in buffer and then filtering with a 0.2 $\mu \mathrm{m}$ filter. Protein concentrations were determined by the adsorbance at $280 \mathrm{~nm}$. The extinction coefficients of lysozyme and $\alpha$-lactalbumin at $280 \mathrm{~nm}$ are 2.63 and $2.04 \mathrm{~L} \mathrm{~g}^{-1} \mathrm{~cm}^{-1}$, respectively.

2.2. Protein Labeling. The fluorescent probes Cy5, Atto 635, Atto 520, and BODIPY FL were acquired from Amersham Biosciences (Uppsala, Sweden), ATTO-TEC (both Atto probes) (Siegen, Germany) and Molecular Probes (Eugene, OR), respectively. The properties of the fluorescent labels used in the experiments are shown in Table 1. A stock solution of 20 $\mathrm{mg} / \mathrm{mL}$ of each dye in DMSO was prepared. The stock solution was divided into $10 \mu \mathrm{L}$ aliquots (containing $0.2 \mathrm{mg}$ of dye) and placed in $1.5 \mathrm{~mL}$ Eppendorf tubes. The dye solutions were kept frozen until use. A lysozyme solution of approximately $10 \mathrm{mg} / \mathrm{mL}$ was prepared by dissolving hen egg white lysozyme in $100 \mathrm{mM}$ sodium carbonate buffer, $\mathrm{pH}$ 9.3. For labeling with Atto 635, $100 \mathrm{mM}$ sodium phosphate, $\mathrm{pH} 7.2$ was used to avoid decomposition of the dye which occurs at higher $\mathrm{pH}$. Approximately $1 \mathrm{~mL}$ of lysozyme solution (10.0 mg lysozyme) was added to an aliquot of dye solution and allowed to mix 30 min end-over-end. Unconjugated dye was separated from the mixture by gel filtration chromatography on a $30 \mathrm{~mL} \mathrm{XK16/20}$ column packed with Sephadex G-15 (Amersham Biosciences) using a flow rate of $3 \mathrm{~mL} / \mathrm{min}$. The gel filtration step also served to exchange the buffer from the dye conjugation buffer to the working buffer for later experiments. The degree of labeling was determined by measuring the absorbance at $280 \mathrm{~nm}$ for lysozyme and at $\lambda_{\mathrm{ex}}$ of the dyes with a Cary $50 \mathrm{Bio}$ UV/Visible spectrophotometer. The absorbance due to the dye at $280 \mathrm{~nm}$ is subtracted using the correction factor $(\mathrm{CF})$ given in Table 1.
The correction factor is defined as the ratio of the dye absorbance at $280 \mathrm{~nm}$ to that at $\lambda_{\mathrm{ex}}$.

2.3. Lysozyme-Dye Conjugate Isocratic Zonal Chromatography. Approximately $1 \mathrm{~mL}$ of SP Sepharose Fast Flow (Amersham Biosciences) was packed into HR 5/5 (5 cm long $\times 0.5 \mathrm{~cm}$ inner diameter) chromatography column. The column was incorporated into an Äkta chromatography system (Amersham Biosciences). The column was equilibrated with at least 5 column volumes of the running buffer. Then $20 \mu \mathrm{L}$ of the purified lysozyme-dye conjugate containing a total protein concentration of approximately $1.5 \mathrm{mg} / \mathrm{mL}$ was injected under isocratic conditions. Only ionic strengths where the mass balances converged are shown. The molar dye/protein (D/P) ratio of the solutions injected varied depending on the efficiency of the labeling reaction for the particular fluorescent label. The retention factor $k^{\prime}=\left(V_{\mathrm{r}}-V_{0}\right) / V_{0}$ was calculated from the retention volume of the protein $\left(V_{\mathrm{r}}\right)$ given by the location of the peak maximum and using retention volume of acetone $\left(V_{0}\right)$ as the accessible volume.

2.4. Confocal Laser Scanning Microscopy. CLSM experiments were performed on a Zeiss LSM510 confocal laser scanning microscope equipped with a C-Apochromat 63x/ $1.2 \mathrm{Wcorr}$ objective lens. The system is equipped with laser excitation sources at 488, 533, and $633 \mathrm{~nm}$ for exciting the fluorophore. A custom-designed flow cell (microcolumn) ${ }^{14}$ containing $50 \mu \mathrm{L}$ of stationary phase was placed on the microscope stage. A $2 \mathrm{mg} / \mathrm{mL}$ protein solution with a molar dye-to-protein ratio $(\mathrm{D} / \mathrm{P})$ of 0.01 was loaded onto the column at a flow rate of $0.05 \mathrm{~mL} / \mathrm{min}$. Images were acquired at either 15 or $30 \mathrm{~s}$ intervals. An experiment with saturated particles and several hundred scans showed that no detectable photobleaching occurs with the fluorophores for the microscope settings used. Settings for the detector gain and laser power were determined for each mobile phase condition by trial and error.

Exchange experiments with unlabeled protein were carried out after the particles were saturated with the $\mathrm{D} / \mathrm{P}=0.01$ protein solution. The feed to the flow cell was simply changed to a 10 $\mathrm{mg} / \mathrm{mL}$ unlabeled solution and the decrease in fluorescence intensity in the particle was observed with the microscope.

Images were exported from the instrument software in TIFF format. Radial concentration profiles were smoothed by averaging all pixels which lie on a concentric circle a fixed distance from the particle center in single pixel radial increments. The particle center in the image was determined by fitting a circle around the edge of the particle by visual inspection.

For the exchange experiments, the relative amount of labeled protein remaining in the particle was determined by integrating the averaged radial intensity profiles to give $Q_{\text {rel }}$, a volumetrically-averaged intensity. $Q_{\text {rel }}(t) / Q_{\text {rel }}(0)$ gives the fraction of labeled protein remaining with $Q_{\text {rel }}$ as defined by Hubbuch et al. ${ }^{14}$

\section{Results and Discussion}

Figure 1 shows representative chromatograms for a $20 \mu \mathrm{L}$ pulse injection of Cy-5-labeled lysozyme on a $1 \mathrm{~mL} \mathrm{SP}$ Sepharose Fast Flow column at pH 7 and $0.5 \mathrm{M}$ ionic strength. The peak maxima are located at different retention volumes for labeled and unlabeled lysozyme even at $0.5 \mathrm{M}$ ionic strength. Chromatograms at other conditions were qualitatively similar to Figure 1. As the ionic strength is lowered, the difference between the retention volume of the labeled lysozyme and that of the unlabeled increases. Retention factors for unlabeled lysozyme and lysozyme labeled with various fluorescent probes are shown in Figure 2. For all fluorescent probes, the retention factor of the dye-protein conjugate differs from that of the 

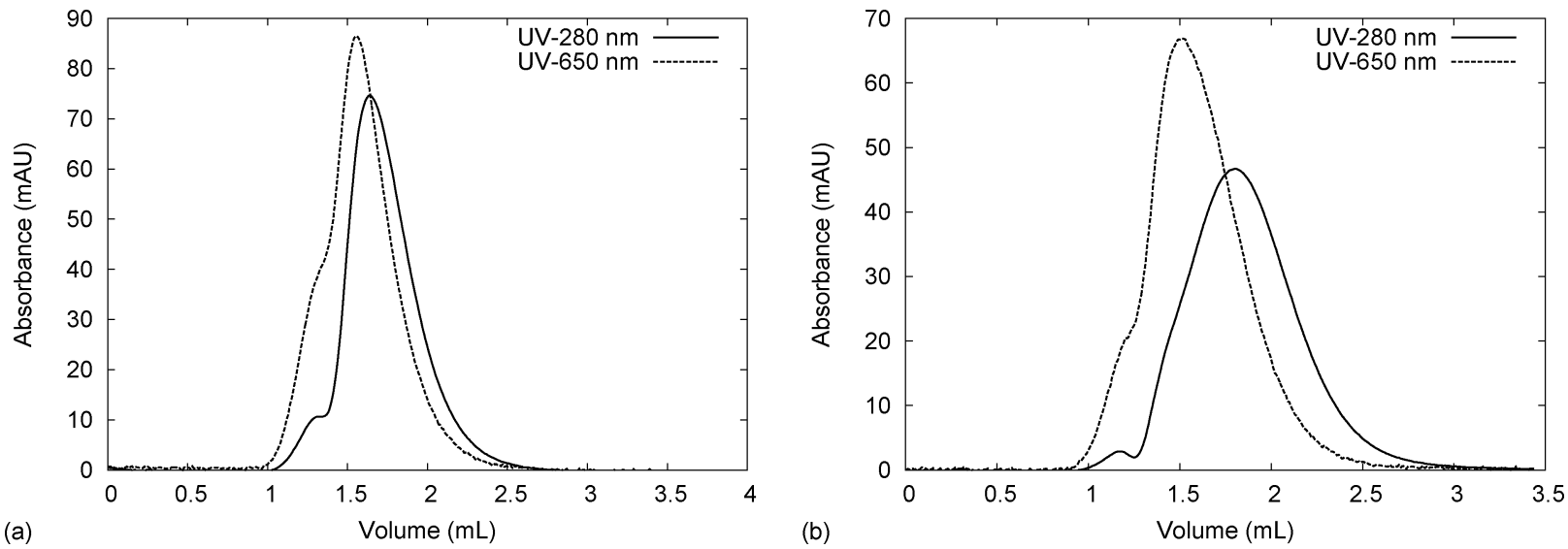

Figure 1. Chromatograms of unlabeled $(280 \mathrm{~nm})$ and Cy5-labeled $(650 \mathrm{~nm})$ lysozyme for a pulse injection on SP Sepharose Fast Flow at pH 7 and 0.5 (a) and $0.4 \mathrm{M}$ (b) ionic strength.

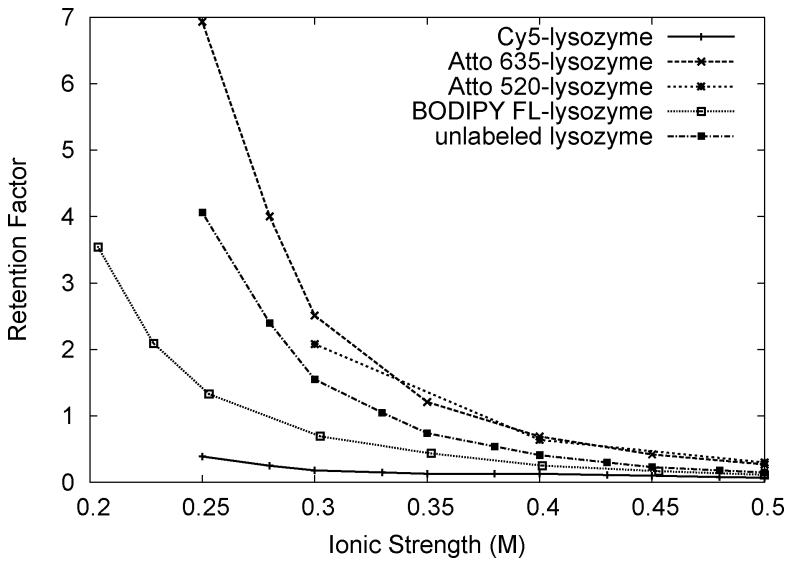

Figure 2. Retention factor $\left(k^{\prime}\right)$ of lysozyme labeled with various fluorescent probes on SP Sepharose FF as a function of ionic strength.

native protein. The charge $(z)$ of the fluorescent probes is listed in Table 1. For positively charged dyes, Atto 635 and Atto 520, the retention factor of the labeled protein is greater than that of the unlabeled protein while protein labeled with the negatively charged dye Cy5 has a smaller retention factor than unlabeled protein. Thus, the effect of the fluorescent label on lysozyme retention is qualitatively consistent with the net charge change of the protein upon labeling. All labels in this study react with the primary amino groups of the protein. Upon conjugation, the reacted amino groups on the protein will no longer be protonated at $\mathrm{pH}$ 7. Thus, in addition to the charge from the label an additional positive charge is lost. Thus, lysozyme labeled with uncharged label BODIPY FL has a retention factor less than native lysozyme which is consistent with the decrease in charge upon conjugation. The same experiments were carried out for $\alpha$-lactalbumin at $\mathrm{pH} 4$ and are shown in Figure 3. The effect of attaching the fluorescent probes on the cation-exchange retention of proteins is very similar for both lysozyme and $\alpha$-lactalbumin. This indicates that the effect of attaching the labels is not a particular property of lysozyme but may be similar for additional proteins.

Stahlberg et al. ${ }^{15}$ has developed a theory of ion-exchange chromatography for the linear region of the adsorption isotherm by solving the Poisson-Boltzmann equation for planar geometry. When the protein surface has a lower surface charge density than the surface of the stationary phase, which is true for many commercial ion-exchange resins, the charge of protein $\left(q_{\mathrm{chr}}\right)$ can be determined from the slope (S) of a plot of $\ln k^{\prime}$ against the inverse square root of ionic strength from $q_{\mathrm{chr}}=\sqrt{S A_{\mathrm{p}} / 135}$

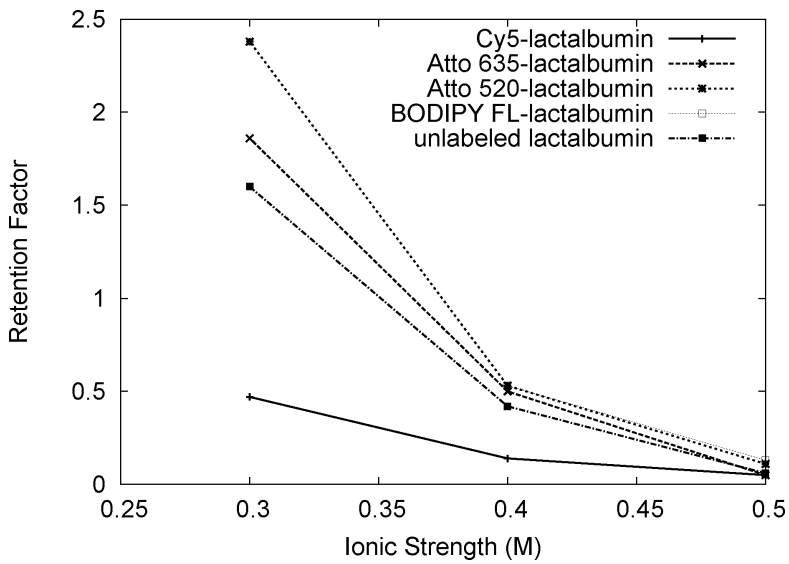

Figure 3. Retention factor of $\alpha$-lactalbumin labeled with various fluorescent probes on SP Sepharose FF as a function of ionic strength. Note: The BODIPY FL and Atto 520 curves are virtually superimposable.

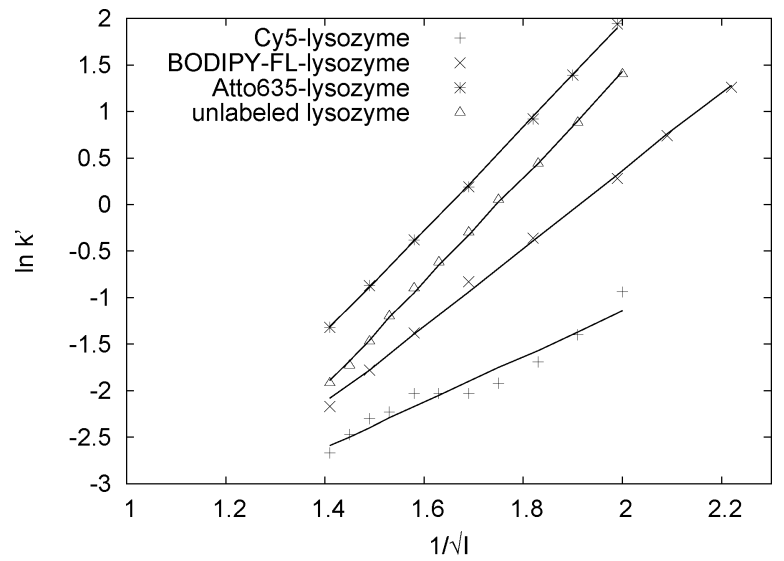

Figure 4. Plot to determine the chromatographic charge $q_{\mathrm{chr}}$ of unlabeled lysozyme and lysoyzme-dye conjugates on SP Sepharose FF, $\mathrm{pH}$ 7. $q_{\mathrm{chr}}$ can be calculated from the slope of the best-fit lines according to the work of Stahlberg et al. ${ }^{15}$ From the slope of the bestfit lines, the charge of the adsorbing species are calculated to be 8.3, 10.7, 12.4, and 12.5 for Cy5-labeled, BODIPY-labeled, Atto635-labeled, and unlabeled lysozyme, respectively.

where $A_{\mathrm{p}}$ is the protein surface area in square angstroms. Figure 4 shows such a plot for unlabeled lysozyme and lysozyme with various fluorescent labels attached. From the slope of the bestfit lines, the charge of the adsorbing species are calculated to be $8.3,10.7,12.4$, and 12.5 for Cy5-labeled, BODIPY-labeled, Atto635-labeled, and unlabeled lysozyme, respectively. The net 


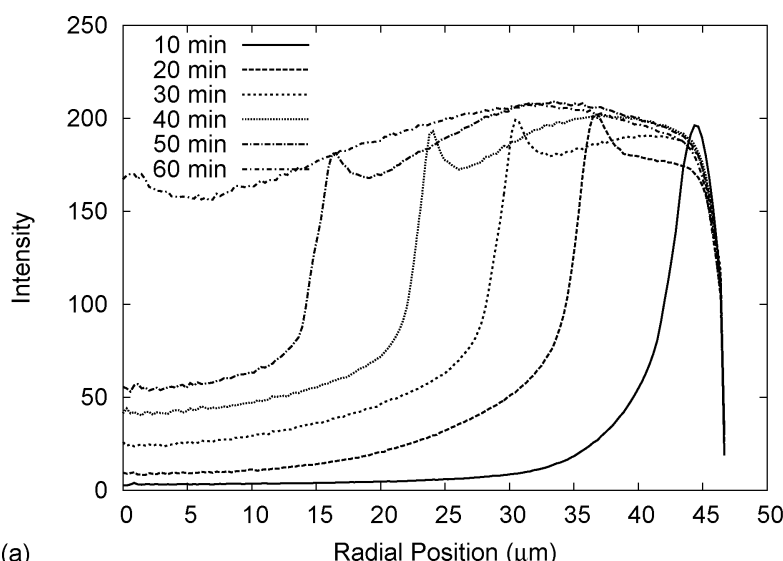

(a)

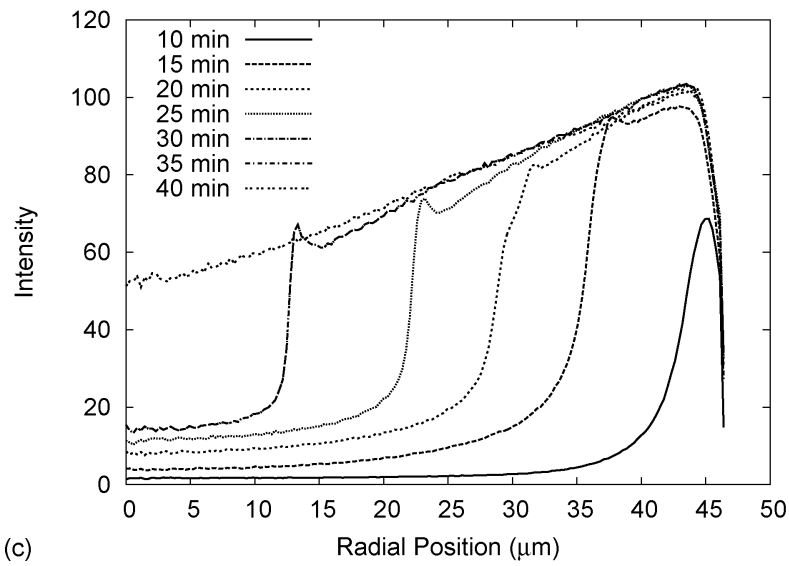

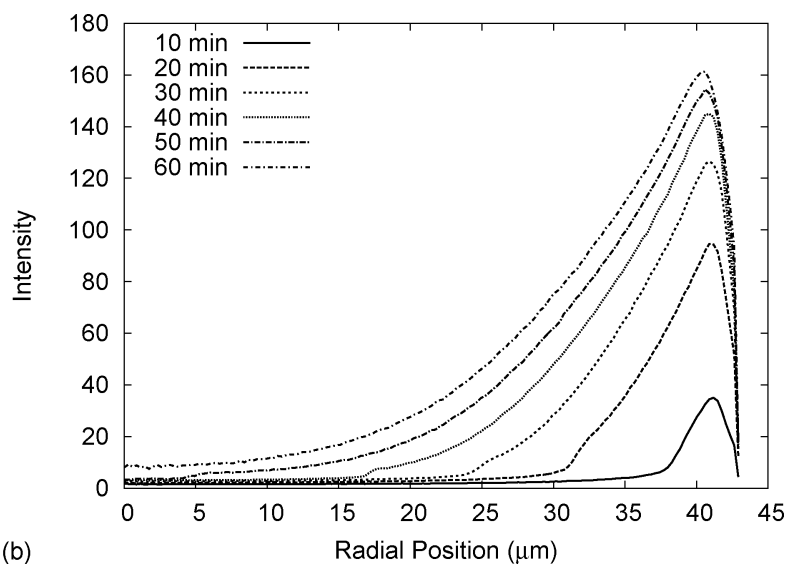

(b)

Figure 5. Intraparticle concentration measured by CLSM using (a) Cy5- (b) Atto 635-, and (c) BODIPY-FL-labeled lysozyme at pH 7, 6 mM ionic strength.

charge of unlabeled lysozyme is slightly overestimated when compared with titration curves at $\mathrm{pH} 7$ which give a net charge of $+8 .{ }^{16}$ The experimental results (on a different stationary phase) analyzed by Stahlberg et al. ${ }^{15}$ also led to an estimate of the lysozyme (chromatographic) net charge which was too high. However, in our study, the estimated differences in net charge between the lysozyme-dye conjugates and unlabeled lysozyme agree fairly well with the charge change expected upon conjugation. It should be pointed out that the fluorescent probes are reacting with primary amino groups which are usually positively charged at $\mathrm{pH} \mathrm{7;} \mathrm{thus,} \mathrm{the} \mathrm{conjugation} \mathrm{reaction} \mathrm{leads}$ to the loss of an additional positive charge. The experimental determined charge differences are slightly more negative than the charge differences predicted from the dye charges listed in Table 1.

We also examined the effect of different fluorescent probes on the intraparticle concentration profiles determined by CLSM. Figures 5-7 show the concentration profiles at various times for Cy5-, Atto 635-, and BODIPY-FL-labeled lysozyme at 6, 50 , and $100 \mathrm{mM}$ ionic strengths.

For the Cy5-labeled lysozyme, a radial hump in the concentration profile is observed at $50 \mathrm{mM}$ as reported previously. ${ }^{4}$ In contrast to the results of Dziennik et al., ${ }^{4}$ we also observe a slight radial hump with Cy5 lysozyme at $6 \mathrm{mM}$ ionic strength. BODIPY-FL-labeled lysoyzme also shows radial humps at 6 and $50 \mathrm{mM}$ ionic strength, but the size of the radial hump is much smaller than that for Cy5. However, for Atto 635-labeled lysozyme, no radial hump is observed in the concentration profiles at any ionic strength. Additionally, for Atto 635-labeled lysozyme, the observed particles do not have a uniform profile even at 60 min while for Cy5 lysozyme, the profile is already nearly uniform. In the case of Atto 635 lysozyme, we believe the center of the particle actually contains the same amount of total protein, but a larger fraction of unlabeled protein than in the case of Cy5 lysozyme. Our data suggest that, for lysozyme, the radial hump previously reported, is caused by the displacement of weaker binding labeled protein by more strongly binding unlabeled protein.

To examine more closely whether displacement was a likely explanation for the formation of the radial hump, labeledunlabeled lysozyme exchange experiments were carried out. These experiments were motivated by similar exchange experiments with polymers ${ }^{17,18}$ A $10 \mathrm{mg} / \mathrm{mL}$ unlabeled lysozyme solution was flowed into a packed bed of stationary phase particles initially in equilibrium with a $2 \mathrm{mg} / \mathrm{mL}$ lysozyme solution $(\mathrm{D} / \mathrm{P}=0.01)$. Equilibrium between fluid-phase and adsorbed proteins is a dynamic process with mobile-phase proteins continually exchanging with adsorbed proteins at the stationary phase surface. The rate of exchange between mobile and bound proteins depends on the affinity of the protein for the stationary phase. For ion-exchange chromatography at fixed $\mathrm{pH}$, it is expected that at higher ionic strength where the binding of the protein to the stationary phase is weaker, the rate of exchange between fluid and adsorbed proteins will be faster than at lower ionic strengths where the protein binds strongly to the stationary phase. It should be pointed out that at 6 and $50 \mathrm{mM}$ ionic strength, washing the loaded stationary phase with lysozyme-free buffer will not lead to measurable desorption of any of the lysozyme dye conjugates in the time frame of the experiments. The presence of unlabeled lysozyme in the mobile phase is necessary for any desorption to occur.

Figure 8 shows the rate of exchange depends on the ionic strength as well as the identity of the fluorescent label. Cy5and BODIPY-FL-labeled lysozyme can be displaced from the 
Protein-Labeling Effects

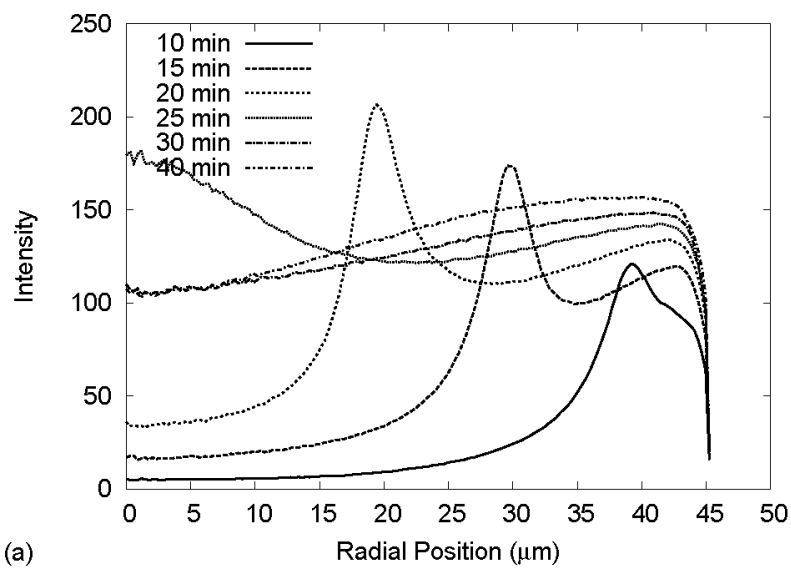

(a)

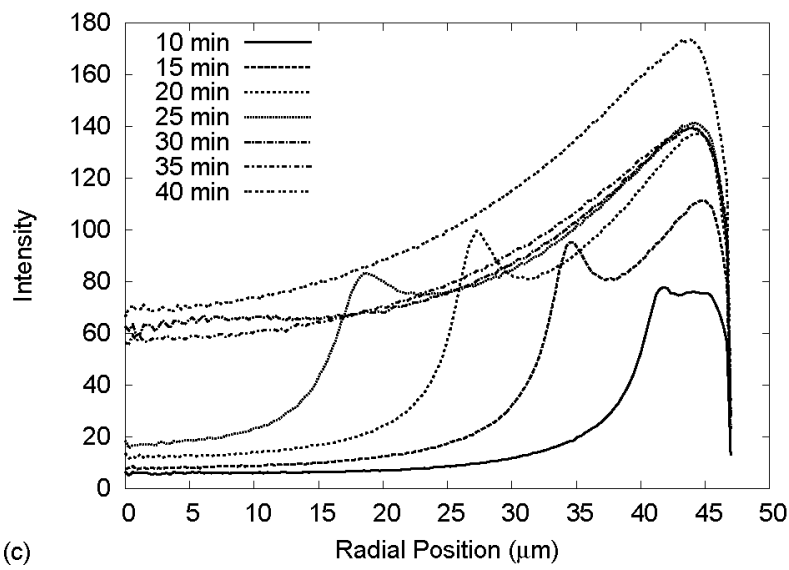

J. Phys. Chem. B, Vol. 109, No. 28, $2005 \mathbf{1 3 8 1 5}$

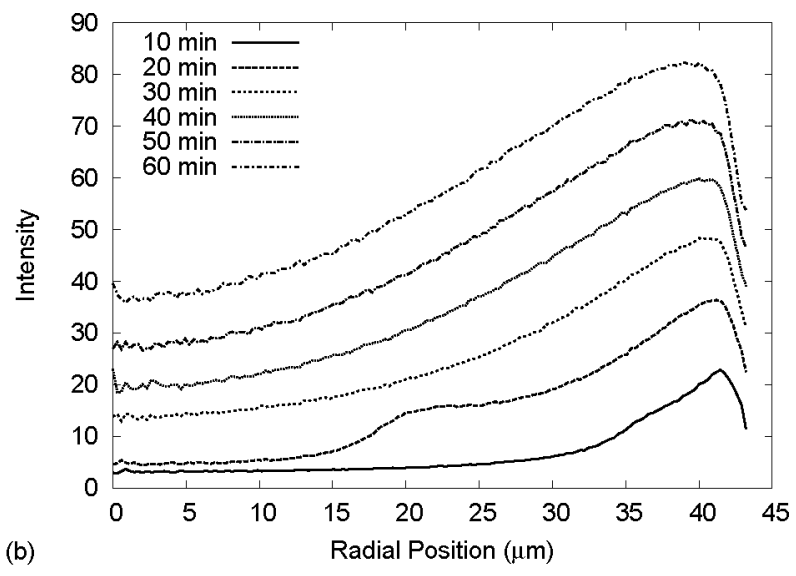

(b)

Figure 6. Intraparticle concentration measured by CLSM using (a) Cy5- (b) Atto 635-, and (c) BODIPY-FL-labeled lysozyme at pH 7, 50 mM ionic strength.
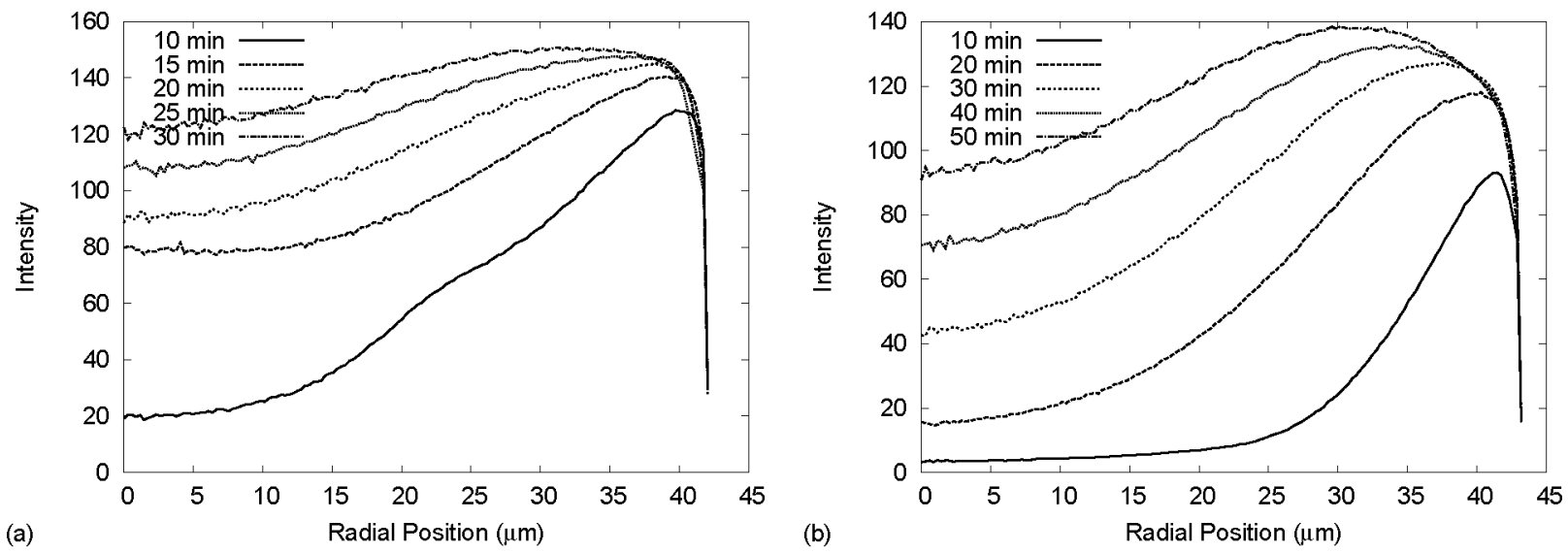

(a)

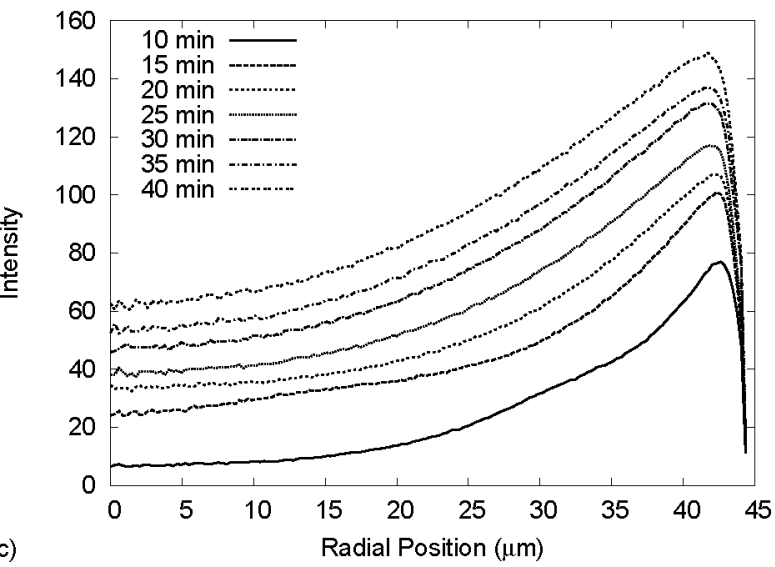

(b)

(c)

Figure 7. Intraparticle concentration measured by CLSM using (a) Cy5- (b) Atto 635-, and (c) BODIPY-FL-labeled lysozyme at pH 7, $100 \mathrm{mM}$ ionic strength. 

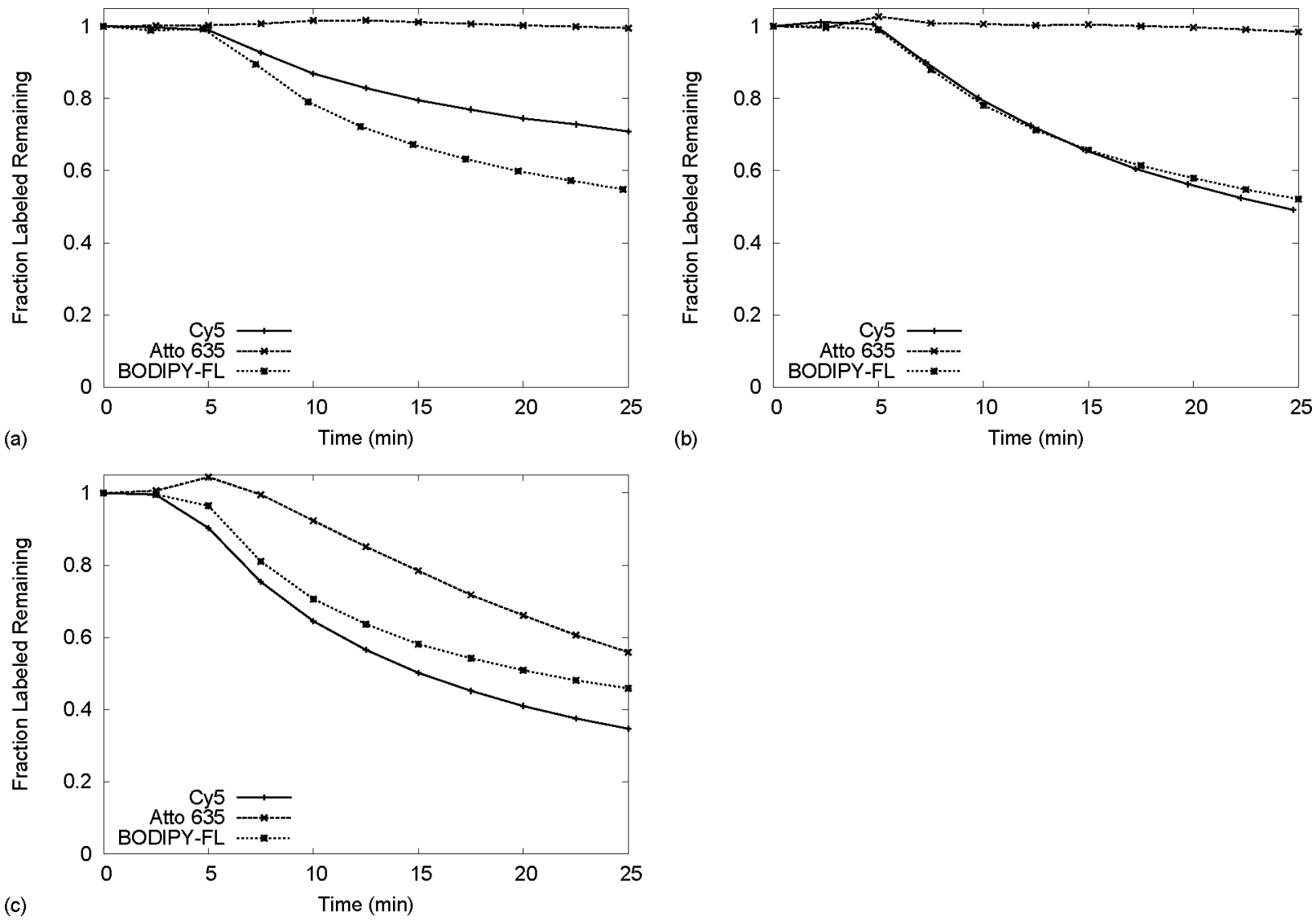

Figure 8. Stationary phase particle initially at equilibrium with a $2 \mathrm{mg} / \mathrm{mL}$ Cy5-lysozyme solution $(\mathrm{D} / \mathrm{P}=0.01)$ at $\mathrm{pH} 7$ and (a) 6 , (b) 50 , and (c) $100 \mathrm{mM}$ ionic strength was subjected to a step input of $10 \mathrm{mg} / \mathrm{mL}$ unlabeled lysozyme at the same $\mathrm{pH}$ and ionic strength and the decrease in fluorescence was monitored. Fraction labeled remaining is the relative amount of labeled protein remaining at the given time on the stationary phase.

stationary phase by unlabeled lysoyzme at all ionic strengths with the rate of exchange increasing as the ionic strength is increased. However, Atto 635-labeled lysozyme cannot be displaced appreciably from the stationary phase at 6 or $50 \mathrm{mM}$ ionic strengths. This is a possible explanation for the lack of a radial hump when lysozyme is labeled with Atto 635.

Our conceptual model of the interior radial hump is as follows: at $6 \mathrm{mM}$ ionic strength, the reduced rate of displacement of Cy5 lysozyme (as compared to $50 \mathrm{mM}$ ionic strength) by unlabeled lysozyme leads to a smaller radial hump. At higher ionic strengths $(100 \mathrm{mM})$, no radial hump is formed because the more diffuse profiles mean there is never a concentrated front of labeled lysozyme which can be displaced by unlabeled lysozyme. Only at intermediate strengths $(50 \mathrm{mM})$ are the conditions balanced appropriately (very steep adsorption fronts along with sufficient ionic strength for rapid displacement) for radial hump formation.

Dziennik et al. ${ }^{4}$ carried out several experiments to rule out artifacts in the experiments due to impurities in the lysozyme source. A few CLSM experiments were also carried out with two different fluorescent labels, Cy5 and FITC, both of which are negatively charged, with varying molar dye/protein ratios. Because FITC is also negatively charged, it is likely that lysozyme-FITC conjugates are more weakly binding than unlabeled lysozyme. The most convincing results from their validation experiments were from multiphoton fluorescence microscopy, which does not require labeling the protein to measure profiles. An extremely limited set of images at $\mathrm{pH} 7$ and $50 \mathrm{mM}$ ionic strength also showed the presence of an interior radial hump. We currently have no explanation for this result in relation to our recent work.
In previous studies, concentration overshoots were also observed with an IgG on SP Sepharose Fast Flow. ${ }^{5,14}$ Data published by Linden et al. ${ }^{3}$ show that $\mathrm{Cy} 3$ and $\mathrm{Cy} 5$ conjugates of a different IgG have slightly shorter retention times than unlabeled IgG. On the basis of the results of the current study and assuming the data for the IgG conjugates used by Linden et al. ${ }^{3}$ are similar to those for the IgG used by Hubbuch et al..$^{5,14}$ which shows the concentration overshoot, this overshoot may also be caused by displacement. Further work will be necessary to determine whether overshoots observed with other proteins ${ }^{4}$ ( $\alpha$-lactalbumin, bovine lactoferrin, and bovine ribonuclease $\mathrm{A}$ ) are also the result of displacement.

\section{Conclusions}

For lysozyme adsorbing on SP Sepharose FF, our results strongly suggest that the radial humps in adsorbed-phase concentration observed in CLSM experiments are an artifact caused by the displacement of weaker-binding labeled lysozyme by more strongly binding unlabeled lysozyme. Modeling work is currently underway to quantitatively describe this phenomenon. Further experiments are necessary to determine whether radial humps reported in the literature for other systems can also be attributed to the displacement or to the electrokinetic transport mechanism proposed by Dziennik et al. ${ }^{4}$ or Liapis et al. $^{6}$

Acknowledgment. The authors acknowledge the financial support of the German Research Society (DFG). We also thank Professor Maria-Regina Kula for valuable discussions and support. 


\section{References and Notes}

(1) Ljunglöf, A.; Hjorth, R. J. Chromatogr. A 1996, 743, 75-83.

(2) Ljunglöf, A.; Thömmes, J. J. Chromatogr. A 1998, 813, 387-395.

(3) Linden, T.; Ljunglöf, A.; Hagel, L.; Kula, M.-R.; Thömmes, J. Sep.

Sci. Technol. 2002, 37, 1-32

(4) Dziennik, S.; Belcher, E.; Barker, G.; DeBergalis, M.; Fernandez,

S.; Lenhoff, A. Proc. Natl. Acad. Sci. U.S.A. 2003, 100, 420-425.

(5) Hubbuch, J.; Linden, T.; Knieps, E.; Ljunglöf, A.; Thömmes, J.; Kula, M.-R. J. Chromatogr. A 2003, 1021, 93-104.

(6) Liapis, A.; Grimes, B. A.; Lacki, K.; Neretnieks, I. J. Chromatogr. A 2001, 921, 135-145. 866.

(7) Schnaible, V.; Przybylski, M. Bioconjugate Chem. 1999, 10, 861-

(8) Grunwaldt, G.; Haebel, S.; Spitz, C.; Steup, M.; Menzel, R. J. Photochem. Photobiol., B 2002, 67, 177-186.

(9) Lewus, R.; Carta, G. AIChE J. 1999, 45, 512-522.
(10) Brooks, C.; Cramer, S. AIChE J. 1992, 38, 1969-1978.

(11) Martin, C.; Iberer, G.; Ubiera, A.; Carta, G. ISPPP 2002, 10-13 November; Heidelberg, Germany, 2002.

(12) Gallant, S. J. Chromatogr. A 2004, 1028, 189-195.

(13) Buschmann, V.; Weston, K.; M.; S. Bioconjugate Chem. 2003, 14 , 195-204.

(14) Hubbuch, J.; Linden, T.; Knieps, E.; Thömmes, J.; M.-R.; K. Biotechnol. Bioeng. 2002, 80, 359-368.

(15) Stahlberg, J.; Jonsson, B.; Horvath, C. Anal. Chem. 1991, 63, 18671874

(16) Kuehner, D.; Engmann, J.; Fergg, F.; Wernick, M.; Blanch, H.; Prausnitz, J. J. Phys. Chem. B 1999, 103, 1368-1374.

(17) Frantz, P.; Granick, S. Phys. Rev. Lett. 1991, 66, 899-902.

(18) Wang, Y.; Rajagopalan, R.; Mattice, W. Macromolecules 1995, $28,7058-7063$ 